\title{
Pre-operative immunotherapy with tumor cryoablation (cryo) plus ipilimumab (ipi) induces potentially favorable systemic and intratumoral immune effects in early stage breast cancer (ESBC) patients
}

\author{
David B Page ${ }^{1 *}$, Adi Diab², Jianda Yuan¹, Zhiwan Dong ${ }^{1}$, Stephen B. Soloman'1, Sujata Patil ${ }^{1}$, Clifford A. Hudis $^{1}$, \\ Jedd D. Wolchok', Larry Norton', Heather L. McArthur ${ }^{1}$
}

From Breast Cancer Immunotherapy Symposium (BRECIS), part of the Sidra Symposia Series, held in partnership with the Society for Immunotherapy of Cancer

Doha, Qatar. 13-14 April 2015

\section{Background}

In mice, cryo plus checkpoint blockade facilitates tumor antigen release, $\mathrm{T}$-cell priming, and improved survival [1]. Here, we assess immune response in ESBC patients using biomarkers that have been attributed to clinical benefit following checkpoint blockade [2-5].

\section{Methods}

Women with ESBC were treated 7-10 days preceding mastectomy with either cryo $(n=6)$, single-dose ipi at $10 \mathrm{mg} / \mathrm{kg}(\mathrm{n}=6)$, or cryo+ipi $(\mathrm{n}=6)$ [6]. From serial blood (baseline \& 1-month post-mastectomy) and tumor (biopsy \& mastectomy), fold-changes following cryo+ipi versus monotherapy were compared (Wilcoxon ranksum) across the following measures: Ki67+ or ICOShi $\mathrm{T}$-cells [2] and intratumoral T-effector/T-regulatory [3] cells by flow cytometry, plasma Th1/Th2 cytokines [4] (Meso Scale Discovery), and intratumoral T-cell expansion by immunohistochemistry [5] and T-cell receptor (TCR) deep sequencing (ImmunoSEQ) [5].

\section{Results}

Cryo+ipi generated greater increases in peripheral Ki67 $+\mathrm{CD} 4+(\mathrm{p}=0.05), \mathrm{Ki} 67+\mathrm{CD} 8+(\mathrm{p}=0.05)$, ICOShiCD4+ $(\mathrm{p}=0.005)$, and ICOShiCD $8+(\mathrm{p}=0.005)$ cells. The

${ }^{1}$ Breast Medicine Service, Memorial Sloan Kettering Cancer Center, New York, New York, USA

Full list of author information is available at the end of the article intratumoral T-effector/regulatory ratio was higher following cryo+ipi, but only when Ki67-gated $(\mathrm{p}=.01)$. Cryo + ipi generated greater increases in IL-2 $(\mathrm{p}=.01)$, IFNI $\hat{I}^{3}$ $(\mathrm{p}=.06)$, and IL-5 $(\mathrm{p}=.09)$. Despite negligible intratumoral changes by immunohistochemistry, cryo+ipi generated more high-magnitude ( 1000 amplicon) clonal expansions by TCR sequencing (medians: 52 v. 3 clones).

\section{Conclusions}

Cryo+ipi is associated with potentially favorable immunologic effects. Ki67-gating and TCR sequencing may identify intratumoral changes otherwise undetectable by flow or IHC.

\footnotetext{
Authors' details

'Breast Medicine Service, Memorial Sloan Kettering Cancer Center, New York, New York, USA. ${ }^{2}$ Department of Melanoma Medical Oncology, MD Anderson Cancer Center, Houston, Texas, USA.

Published: 14 August 2015

References

1. Waitz R, Solomon SB, Petre EN, Trumble AE, Fasso M, Norton L, Allison JP: Potent induction of tumor immunity by combining tumor cryoablation with anti-CTLA-4 therapy. Cancer research 2012, 72:430-9.

2. Di Giacomo AM, Calabro L, Danielli R, Fonsatti E, Bertocci E, Pesce I, Fazio C, Cutaia O, Giannarelli D, Miracco C, Biagioli M, Altomonte M, Maio M: Longterm survival and immunological parameters in metastatic melanoma patients who responded to ipilimumab $10 \mathrm{mg} / \mathrm{kg}$ within an expanded access programme. Cancer Immunol Immunother 2013, 62:1021-8.
} 
3. Hodi FS, Butler M, Oble DA, Seiden MV, Haluska FG, Kruse A, Macrae S, Nelson M, Canning C, Lowy I, Korman A, Lautz D, Russell S, Jaklitsch MT, Ramaiya N, Chen TC, Neuberg D, Allison JP, Mihm MC, Dranoff G: Immunologic and clinical effects of antibody blockade of cytotoxic $T$ lymphocyte-associated antigen 4 in previously vaccinated cancer patients. Proc Natl Acad Sci USA 2008, 105:3005-10.

4. Yuan JOF, Jefferson M, Li H, Gallardo H, Ku G, Wolchok J, Scher H, Allison J, Slovin S: Cytokine changes in castrate metastatic prostate cancer (CPMC) patients (pts) treated with ipilimumab (Ipi). 2009 ASCO Annual Meeting Orlando, FL; 2009.

5. Tumeh PC, Harview CL, Yearley JH, Shintaku IP, Taylor EJ, Robert L, Chmielowski B, Spasic M, Henry G, Ciobanu V, West AN, Carmona M, Kivork C, Seja E, Cherry G, Gutierrez AJ, Grogan TR, Mateus C, Tomasic G, Glaspy JA, Emerson RO, Robins H, Pierce RH, Elashoff DA, Robert C, Ribas A: PD-1 blockade induces responses by inhibiting adaptive immune resistance. Nature 2014, 515:568-71.

6. Diab A, Solomon SB, Comstock C, Maybody M, Sacchini V, Durack JC, Blum B, Yuan J, Patil S, Neville DA, Sung JS, Kotin A, Morris EA, Brogi E, Morrow M, Wolchok JD, Allison JP, Hudis CA, Norton L, MCArthur HL: A pilot study of preoperative (Pre-op), single-dose ipilimumab (Ipi) and/or cryoablation (Cryo) in women (pts) with early-stage/resectable breast cancer (ESBC). 2013 ASCO Breast Cancer Symposium; 2013 September 7-9 San Francisco, CA.; 2013.

doi:10.1186/2051-1426-3-S1-O6

Cite this article as: Page et al:: Pre-operative immunotherapy with tumor cryoablation (cryo) plus ipilimumab (ipi) induces potentially favorable systemic and intratumoral immune effects in early stage breast cancer (ESBC) patients. Journal for ImmunoTherapy of Cancer 2015 3(Suppl 1):06.

\section{Submit your next manuscript to BioMed Central} and take full advantage of:

- Convenient online submission

- Thorough peer review

- No space constraints or color figure charges

- Immediate publication on acceptance

- Inclusion in PubMed, CAS, Scopus and Google Scholar

- Research which is freely available for redistribution

Submit your manuscript at www.biomedcentral.com/submit 